\title{
The Study of Pyrolytic Products of Polymer Materials by Mass Spectroscopy (VII) * The Combustion Gas from Resin Laminated Board in Fire
}

\author{
Kiyoshi Hamano and KinjI Hiramatsu
}

(Received 20 May 1970)

\section{Introduction}

Recently, by the increasing use of non-flammable and fireproof material in constructions, more attentions must be paid to the injurious gas and smoke in fire rising from the interia materials. It is not clear, on the other hand, that how much the injurious gas and smoke rises in fire from the material. In order to get information for the production of the nonflammable board and the board which do not cause the hazardous gas in fire, the combustion gas and the smoke of about 13 of the commercially available resin laminated boards were analysed. This paper deals with the composition of the combustion gas component and the smoke concentration in the fire of these interia materials.

\section{Experimental}

\subsection{Instrument}

a) Analytical Instrument

A RMU-5B (Hitachi) mass spectrometer was used for the analyses of the combustion gases. The operating conditions were as follows: The ion accelelating voltage was $2 \mathrm{KV}$ and ion current was $100 \mu \mathrm{A}$ with ionization chamber kept at about $250{ }^{\circ} \mathrm{C}$. The analysed area was from m/e 12 to 150 and m/e 2 .

b) The other Analytical method Gas detection tube (Komyo Chemical Co.) was used for detection of hydrogen cyanide.

\section{2 The Combustion Furnace}

In order to minimize the variation of the analytical results, the combustion gas was treated in much larger quantities. Fig. 1 shows combustion furnace which was improved in this study. This instrument is the combustion furnace prescribed in JIS Z 2150. The volume of the chamber is about $50 \mathrm{l}$ and a box of volume of $125 \mathrm{l}$ is attached on the chimney to collect the smoke. The total volume of the combustion chamber and the collecting box is about $175 \mathrm{l}$. The concentration of the smoke in the box was determined by electric photometer. The electric heater is set instead of LPG burner as the fire source, and when the temperature upon the heater was kept at $600^{\circ} \mathrm{C}$, the sample was introduced and burned.

2. 3 The preparation of the sample

The preparation of the sample was as follows:

The sample was dried at $50 \pm 2{ }^{\circ} \mathrm{C}$ for $48 \mathrm{hrs}$ and kept with silica gel in a

* Industrial Research Institute, Osaka Prefecture Enokojima Kamino-cho, Nishi-ku, Osaka, Japan 
The Study of Pyrolytic Products of Polymer Materials by Mass Spectroscopy( VI) The Combustion Gas from Resin Laminated Board in Fire

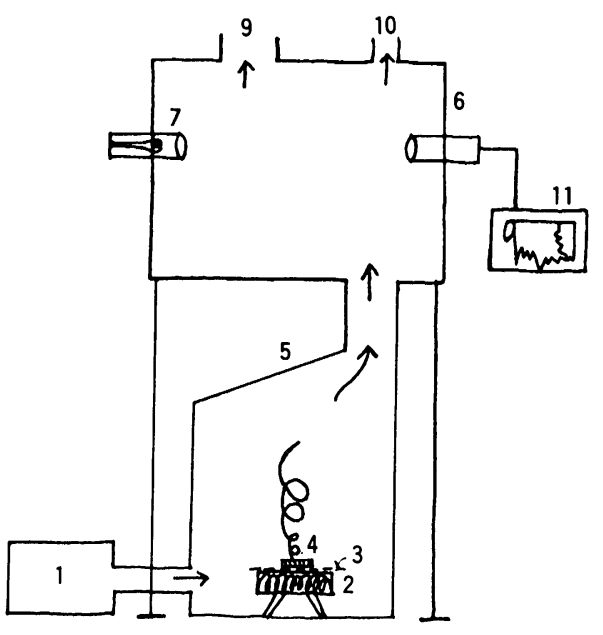

Fig. 1. The Combustion furnace and the instrument for measuring the smoke concentration.

1. Compressor for the inlet of the air (attached'a gas flowmeter)

2: Electric heater $600 \mathrm{w}$

3: Stainless wire net

4: Sample

5: Combustion chamber (JIS. Z. 2150)

6: Measuring chamber for the smoke

7: Light Source for the measurement of the smoke concentration.

8: Electric photometer (CdS)

9: Exhaust gas pipe

10: Sampling pipe for the Combustion gas.

11: Recorder

desiccater.

\section{4 Gas sampling}

After the temperature of the atomosphere on the heater was rised up to about $500{ }^{\circ} \mathrm{C}$ some quantities of the sample material was placed on the stainless steel wire net, and allowed to burn.

The combustion gas was collected from gas sampling pipe into a sampling bottle which was previously evacuated below $10^{-3} \mathrm{mmHg}$. The gas sampling was made at 5 minutes and 10 minutes respectively, after the combustion took place.

2. 5 The combustion states of samples
After the combustion was started, white smoke rises violently for 1 to 3 minutes, the appearance of the sample got into dark brown. Occasionaly, flushover was observed in this period. In this case, the smoke concentration was decreased temporarily and then increased.

The maximum concentration was obtained after 3 to 5 minutes and it decreased gradually. After the combustion was over, tarry materials were left on the furnacing chamber and ash was remained on the heater.

\section{Results and Discussion}

It is known that the combustion temperature of the resin laminated board is about $500^{\circ} \mathrm{C}$, hence the samples were burned in a tightly closed chamber at about $500^{\circ} \mathrm{C}$, in this study. The intensity of the smoke during the combustion was recorded by the electricphotometer. The composition of the combustion gas was analysed by means of mass spectrometry. The components of the gas which can not be analysed by the mass spectrometry were determined by the other method. The samples used in this study are listed in Table 1 .

\section{1 Analysis of combustion gas by the mass spectrometry}

Figs. 2-14 show the spectra of the combustion gas of the resin laminated board. The spectra of these samples show a similar pattern. The analysis of the mass spectra was made as usual, starting from higher mass peaks. On assuming that the peak, m/e 44 was resulted only from carbon dioxide, the peak hight of m/e 12 was too high to be due to carbon dioxide. Moreover, the intensity of the peak at 28 was also overcame to be resulted from carbon dioxide only. These observations together' with the 


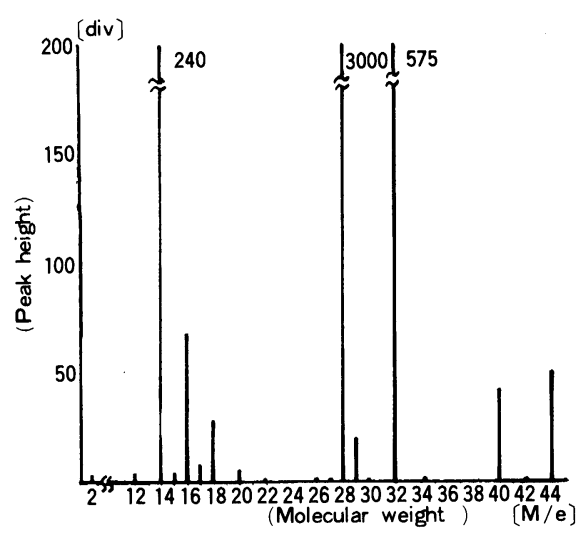

Fig. 2. Mass spectrum of the combustion gas (Sample No. 1) Air: $51 / \mathrm{min}$

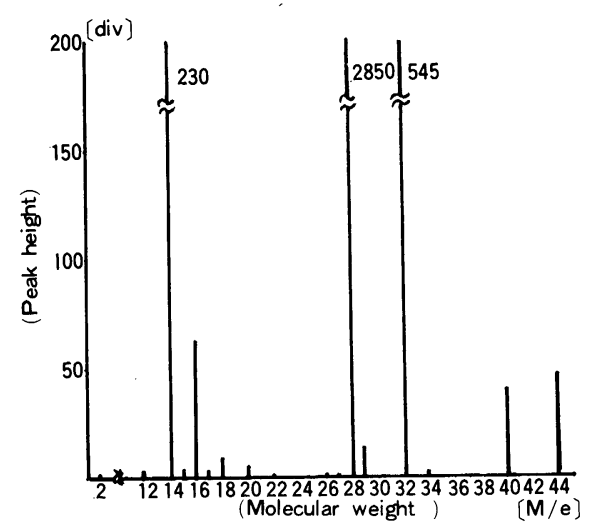

Fig. 4. Mass spectrum of the combustion gas (Sample No. 3) Air: 51/min

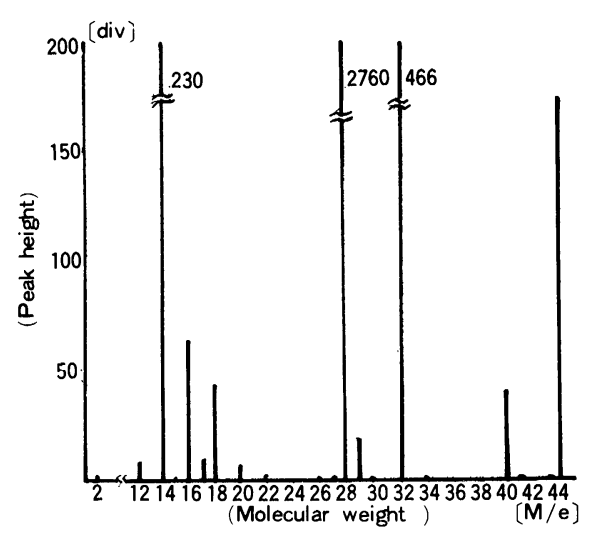

Fig. 6. Mass spectrum of the combustion gas (Sample No.5) Air: $51 / \mathrm{min}$

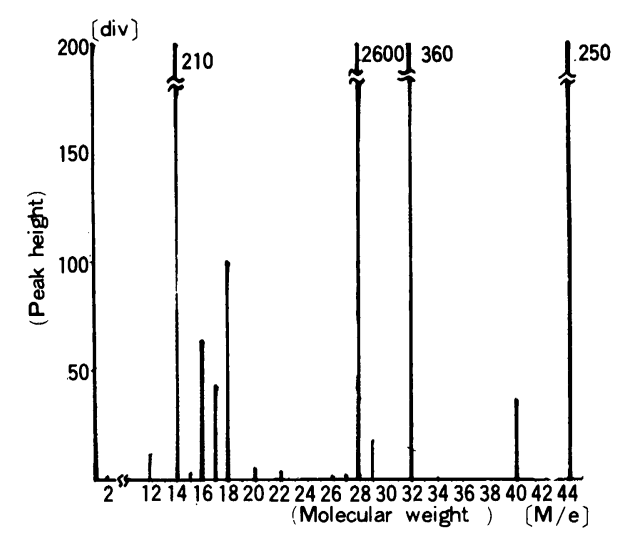

Fig. 3. Mass spectrum of the combustion gas (Sample No. 2) Air: $51 / \mathrm{min}$

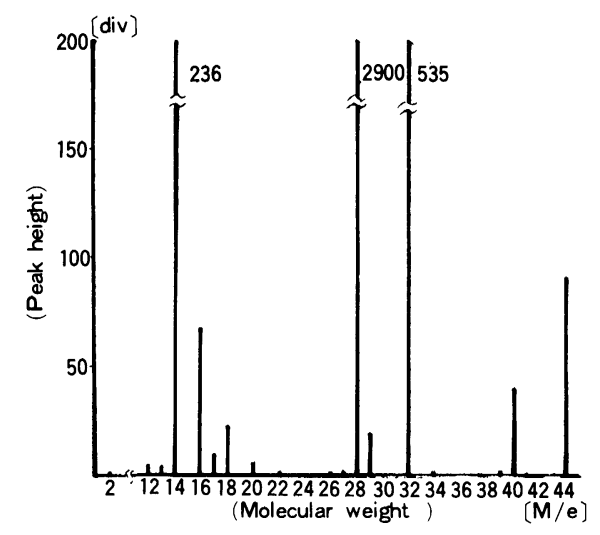

Fig. 5. Mass spectrum of the combustion gas (Sample No.4) Air: $51 / \mathrm{m}$ in

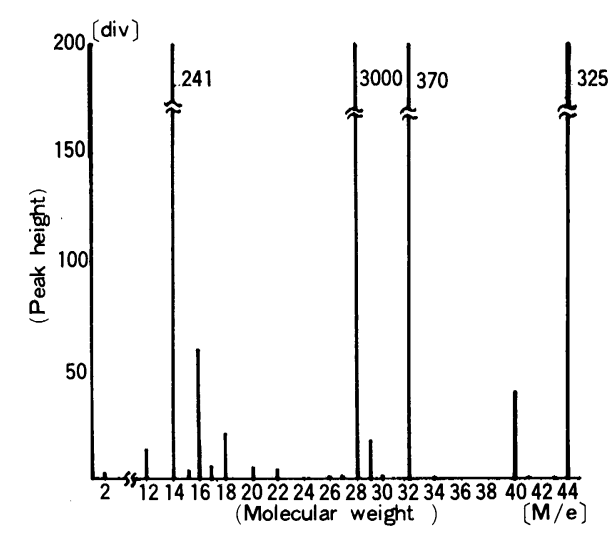

Fig. 7. Mass spectrum of the combustion gas (Sample No.6) Air: $51 / \mathrm{min}$ 
The Study of Pyrolytic Products of Polymer Materials by Mass Spectroscopy (VI)

The Combustion Gas from Resin Laminated Board in Fire

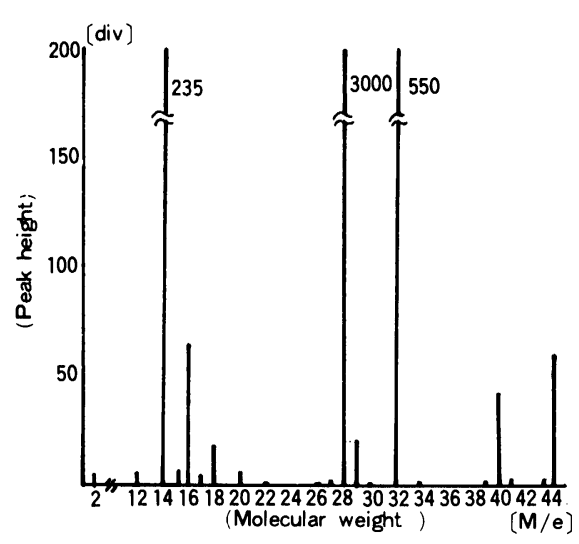

Fig. 8. Mass spectrum of the combustion gas (Sample No. 7) Air: $51 / \mathrm{min}$

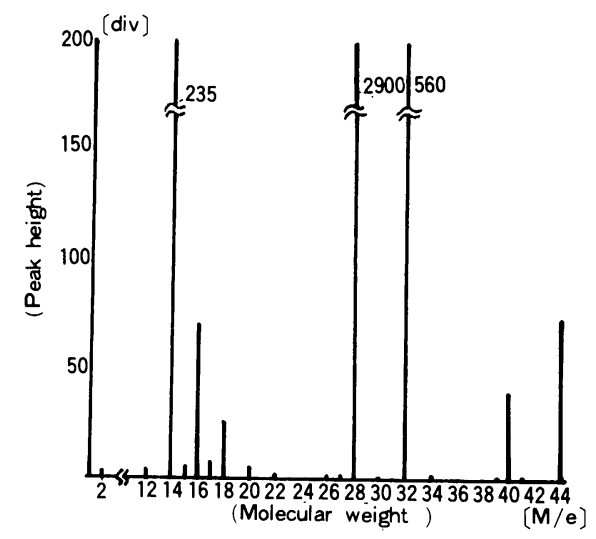

Fig. 10. Mass spectrum of the combustion gas (Sample No.9) Air: $51 / \mathrm{min}$

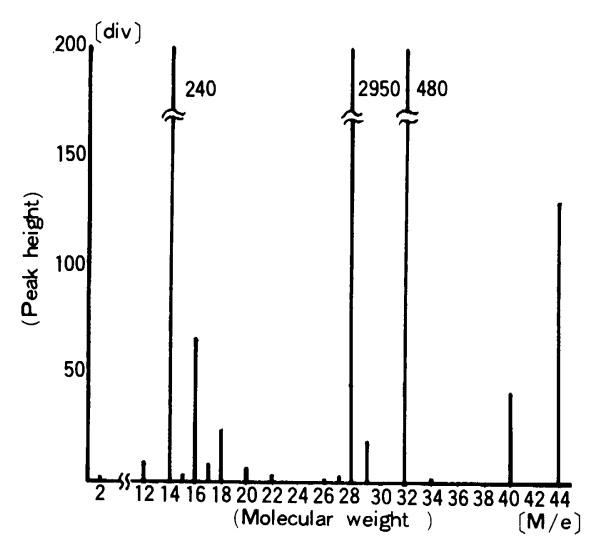

Fig. 12. Mass spectrum of the combustion gas (Sample No. 11) Air: $51 / \mathrm{min}$

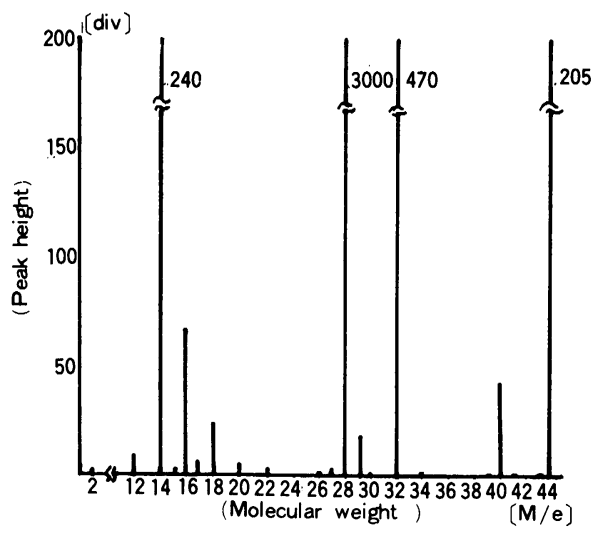

Fig. 9. Mass spectrum of the combustion gas (Sample No. 8) Air: $51 / \mathrm{min}$

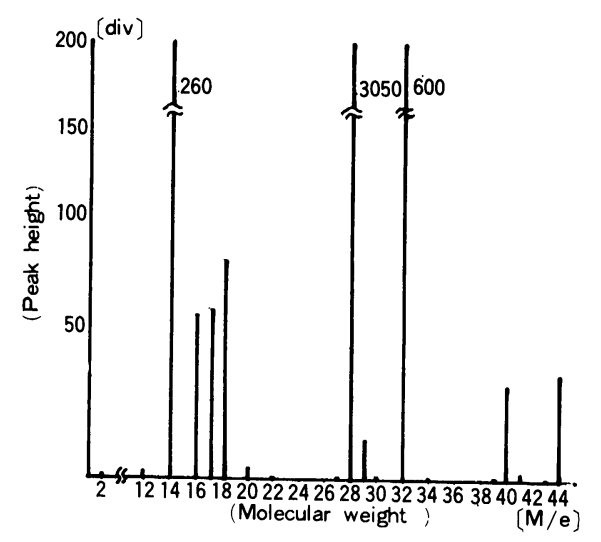

Fig. 11. Mass spectrum of the combustion gas (Sample No. 10) Air: $51 / \mathrm{min}$

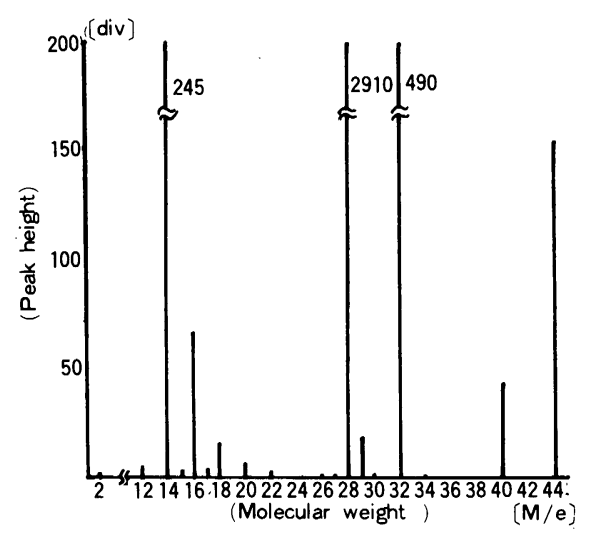

Fig. 13. Mass spectrum of the combu tion gas (Sample No. 12) Air: $51 / \mathrm{min}$ 


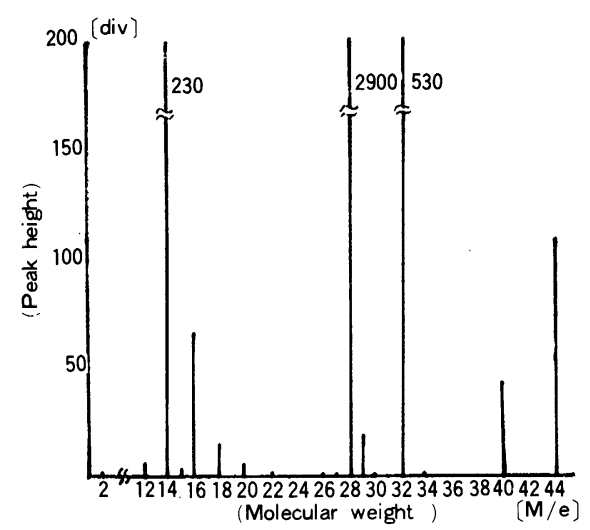

Fig. 14. Mass spectrum of the combustion gas (Sample No.13) Air: $51 / \mathrm{min}$

existance of the peak at $\mathrm{m} / \mathrm{e} 14$, suggest that the existance of nitrogen and carbon monoxide in the combustion gas. The amount of oxygen was determined from the residual peak height after substraction of the contribution of the carbon dioxide at the mass number $\mathrm{m} / \mathrm{e} 32$.

Propane and the propylene were determined by the peak height of mass number 41 and 42 , respectively. The carbon dioxide content was determined from the residual peak height after substruction of the contribution of the propane at the mass number 44 . The content of ethane and ethylene was determined from the peak height of mass number 21 and 27 . From the residual peak height of mass number 14 and 28 , the contents of carbon monoxide and nitrogen were determined. The content of hydrogen was determined from the mass number 2 .

The error of the analysis seemed to be due to the moisture and the complexity of the gas. The mass spectra which were obtained by using larger amount of samples showed a plenty of smaller peaks between the mass number from 96 to 45 . These were $\mathrm{m} / \mathrm{e} 92,91,82,81,78$, $72,68,60$ and 58 to 50 . The peak heights of $\mathrm{m} / \mathrm{e} 91$ and 92 were characteristic; the peak height of $\mathrm{m} / \mathrm{e} 91$ was higher than that of 92 . From these observation, toluene was considered to be present in the sample. Methyl furane was elucidated from the peak of the $\mathrm{m} / \mathrm{e} 82$, and this compound was considered to be produced by the demethylation of dimethyl furane. Benzene was also elucidated from the peak of mass number 78 and from 58 to 50 , and also from mass number 72. Methyl ethyl ketone was also detected. From the other smaller peak, it was concluded that hydrocarbon of C-4, ethyl alcohol, and acetone were present although the amounts were small.

Tables $1-10$ show the components of the combustion gas, obtained from the combustion of $100 \mathrm{~g}$ of resin laminated board in the combustion chamber.

Tables 2-4 show the components of the combustion gas when the combustion was made under usual atmospher, and Tables $5-7$ show the results obtained with blowing air, $11 / \mathrm{min}$ into the chamber. Tables $8-10$ show that of $5 \mathrm{l} / \mathrm{min}$. In all cases spectra were almost identical to each other therefore the main product gas seemed to be contained the identical components.

The main gaseous components were carbon dioxide, argon, oxygen, nitrogen, carbon monoxide, methane and hydrogen. Among the other smaller amount of components, hydrogen cyanide, benzene and toluene were detected. Ethane and ethylene were also detected when the combustion was carried out without blowing additional air into the combustion chamber.

3. 2 The smoke concentration

There are "filter paper method", "weighting method", "counting particles 
The Study of Pyrolytic Products of Polymer Materials by Mass Spectroscopy (VII)

The Combustion Gas from Resin Laminated Board in Fire

method" and "coefficient of decreasing light method" for the measurment of the smoke concentration. The latter method is used in fire. In the present study, the area of the surface of the sample was different to the samples, it was difficult to apply the coefficient of the decreasing light to the present study, hence the transmittance of light are shown in Figs. 15-27. When the flushover was occured, decreasing concentration was resulted for the time being.

Table 1. Sample material

\begin{tabular}{|c|c|c|}
\hline $\begin{array}{c}\text { Sample } \\
\text { No. }\end{array}$ & Adhesive agent & Surface finishing materials \\
\hline 1 & urea resin & polyvinyl acetate resin, urea resin, paper, aminoalkyd resin \\
\hline 2 & urea resin & polyacryl resin, polyphthalate resin, aminoalkyd resin \\
\hline 3 & urea resin & polyacryl resin, polyphthalate resin, aminoalkyd resin \\
\hline 4 & urea resin & polyacryl resin, polyphthalate resin, aminoalkyd resin \\
\hline 5 & urea resin & pohyvinyl acetate, melamine resin, polyester resin \\
\hline 6 & urea resin & polyacryl emulsion resin, polyvinyl chloride sheet $(0.07 \mathrm{~m} / \mathrm{m})$ \\
\hline 7 & phenolic resin & polyphthalate resin \\
\hline 8 & melamine resin & polyacryl resin, polyurethane resin. \\
\hline 9 & melamine resin & polyacryl resin polyurethane resin \\
\hline 10 & urea resin & polyvinyl acetate resin, melamine resin, paper, aminoalkyd resin \\
\hline 11 & urea resin & - \\
\hline 12 & urea resin & - \\
\hline 13 & urea resin & polyacryl resin, polyphthalate resin, aminoalkyd resin \\
\hline
\end{tabular}

Table 2. The components of the combustion gases (Ol/min.)

\begin{tabular}{|c|c|c|c|c|c|c|c|c|c|c|c|c|c|c|}
\hline $\begin{array}{c}\text { Sample } \\
\text { No. }\end{array}$ & $\begin{array}{l}\text { Time } \\
\text { (min.) }\end{array}$ & $\mathrm{CO}_{2}(\%)$ & $\left.\mathrm{C}_{3} \mathrm{H}_{4} \%{ }^{\prime}\right)$ & $\mathrm{C}_{3} \mathrm{H}_{6}(\%)$ & $\operatorname{tr}^{\prime} \%$ & $0_{2}(\%)$ & $\mathrm{C}_{2} \mathrm{H}_{6}(\%)$ & $\mathrm{C}_{2} \mathrm{H}_{4}(\%)$ & $\mathrm{N}_{2}(\%)$ & $\mathrm{CO}(\%)$ & $\mathrm{CH}_{4}\{\%\}$ & $\mathrm{H}_{2}(\%)$ & HCS $(\mathrm{PP})$ & \\
\hline \multirow[t]{3}{*}{1} & 5 & 5.0 & 0.0 & 0.0 & 0.7 & 14.9 & 0.0 & 0.0 & 71.5 & 0.4 & 0.1 & 0.1 & 65 & \multirow{3}{*}{$\begin{array}{l}\text { Benzene. toluenc and the } \\
\text { compounds haring molecular } \\
\text { weight of } 36 \text { and } 64 \text { are } \\
\text { detected in small amount. }\end{array}$} \\
\hline & 10 & 6.2 & 0.0 & 0.0 & 0.7 & 13.6 & 0.1 & 0.1 & 71.5 & 0.8 & 0.2 & 0.3 & 80 & \\
\hline & 15 & 8.7 & 0.0 & 0.0 & 0.7 & 11.0 & 0.1 & 0.1 & 71.0 & 1.3 & 0.2 & 0.3 & 98 & \\
\hline \multirow[t]{3}{*}{2} & 5 & 5.9 & 0.0 & 0.0 & 0.7 & 13.8 & 0.0 & 0.0 & 72.0 & 0.5 & 0.1 & 0.1 & 105 & \multirow{3}{*}{$\begin{array}{l}\text { Benzene, toluenc and the } \\
\text { compounds having molecular } \\
\text { weight } 56 \text { and } 82 \text { are } \\
\text { detected in small amount }\end{array}$} \\
\hline & 10 & 7.5 & 0.0 & 0.0 & 0.7 & 12.5 & 0.0 & 0.1 & 71.0 & 1.0 & 0.1 & 0.2 & 120 & \\
\hline & 15 & 9.5 & 0.0 & 0.0 & 0.7 & 10.4 & 0.0 & 0.1 & 70.0 & 1.5 & 0.1 & 0.2 & 150 & \\
\hline \multirow[t]{3}{*}{3} & 5 & 1.2 & 0.0 & 0.0 & 0.7 & 19.0 & 0.0 & 0.0 & 70.0 & 0.6 & 0.1 & 0.1 & 5) & \multirow{3}{*}{ " } \\
\hline & 10 & 2.2 & 0.0 & 0.0 & 0.7 & 18.2 & 0.1 & 0.1 & 69.2 & 0.8 & 0.2 & 0.2 & $60)$ & \\
\hline & 15 & 3.2 & 0.0 & 0.0 & 0.7 & 16.9 & 0.1 & 0.1 & 70.0 & 0.9 & 0.2 & 0.2 & 75 & \\
\hline \multirow[t]{3}{*}{4} & 5 & 5.2 & 0.0 & 0.0 & 0.7 & 14.9 & 0.1 & 0.1 & 70.0 & 0.5 & 0.1 & 0.1 & 35 & \multirow{3}{*}{ " } \\
\hline & 10 & 6.6 & 0.0 & 0.0 & 0.7 & 13.4 & 0.1 & 0.1 & 70.5 & 0.8 & 0.2 & 0.2 & 65 & \\
\hline & 15 & 7.6 & 0.0 & 0.0 & 0.7 & 12.4 & 0.1 & 0.1 & 69.8 & 1.3 & 0.2 & 0.2 & 85 & \\
\hline \multirow[t]{3}{*}{5} & 5 & 5.9 & 0.0 & 0.0 & 0.7 & 13.9 & 0.0 & 0.1 & 70.6 & 0.4 & 0.0 & 0.1 & 48 & \multirow{3}{*}{$\begin{array}{l}\text { Benzene, toluene and the } \\
\text { compounds haring molecular } \\
\text { weight } 56 \text {. and } 66 \text { are } \\
\text { detected in small amount }\end{array}$} \\
\hline & 10 & 7.6 & 0.0 & 0.0 & 0.7 & 12.6 & 0.0 & 0.1 & 69.5 & 1.2 & 0.3 & 0.2 & 40) & \\
\hline & 15 & 8.6 & 0.0 & 0.0 & 0.7 & 11.3 & 0.0 & 0.1 & 70.0 & 1.3 & 0.3 & 0.1 & 30 & \\
\hline
\end{tabular}




\section{K. Hamano and K. Hiramatsu}

Table 3. The components of the combustion gases $(01 / \mathrm{min}$.)

\begin{tabular}{|c|c|c|c|c|c|c|c|c|c|c|c|c|c|c|}
\hline $\begin{array}{c}\text { Sample } \\
\text { No. }\end{array}$ & $\begin{array}{c}\text { Time } \\
(\text { min. })\end{array}$ & $\mathrm{CO}_{2}(\%)$ & $\mathrm{C}_{3} \mathrm{H}_{8}(\%)$ & $\mathrm{C}_{3} \mathrm{H}_{6}(\%)$ & $\operatorname{Ar}(\%)$ & $\mathrm{O}_{2}(\%)$ & $\mathrm{C}_{2} \mathrm{H}_{6}(\%$ & $\mathrm{C}_{2} \mathrm{H}_{4}(\%)$ & $N_{2}(\%)$ & $\mathrm{C} 0(\%)$ & $\mathrm{CH}_{4}(\%)$ & $\mathrm{H}_{2}(\%)$ & $\mathrm{HCN}(\mathrm{PPm})$ & \\
\hline \multirow[t]{3}{*}{6} & 5 & 5.4 & 0.0 & 0.0 & 0.7 & 14.7 & 0.0 & 0.0 & 72.0 & 0.3 & 0.0 & 0.1 & 75 & \multirow{3}{*}{$\begin{array}{l}\text { Benzene, toluene and the } \\
\text { compounds having molecular } \\
\text { weight } 56 \text { and } 64 \text { are } \\
\text { detected in small amount }\end{array}$} \\
\hline & 10 & 6.4 & 0.0 & 0.0 & 0.7 & 13.4 & 0.1 & 0.1 & 71.0 & 0.7 & 0.2 & 0.2 & 90 & \\
\hline & 15 & 6.7 & 0.0 & 0.0 & 0.7 & 12.9 & 0.1 & 0.1 & 70.0 & 0.5 & 0.2 & 0.2 & 130 & \\
\hline \multirow[t]{3}{*}{7} & 5 & 7.6 & 0.0 & 0.0 & 0.7 & 12.8 & 0.0 & 0.1 & 69.0 & 0.6 & 0.2 & 0.1 & 35 & \multirow{3}{*}{$\begin{array}{l}\text { Benzene, toluene and the } \\
\text { compound having molecular } \\
\text { weight } 56 \text { is deteected in } \\
\text { small amount }\end{array}$} \\
\hline & 10 & 9.5 & 0.0 & 0.0 & 0.7 & 10.5 & 0.1 & 0.1 & 68.5 & 1.4 & 0.3 & 0.5 & 25 & \\
\hline & 15 & 9.8 & 0.0 & 0.0 & 0.7 & 9.4 & 0.0 & 0.1 & 72.0 & 1.2 & 0.3 & 0.6 & 20 & \\
\hline \multirow[t]{3}{*}{8} & 5 & 5.6 & 0.0 & 0.0 & 0.7 & 14.3 & 0.1 & 0.1 & 70.0 & 0.3 & 0.0 & 0.1 & 90 & \multirow{3}{*}{$\begin{array}{l}\text { Benzene, toluene and the } \\
\text { compounds having molecular } \\
\text { weight } 82 \text { and } 56 \text { are } \\
\text { detected in smaller amount } \\
\text { than the other sample }\end{array}$} \\
\hline & 10 & 6.9 & 0.0 & 0.0 & 0.7 & 12.7 & 0.1 & 0.1 & 70.0 & 0.4 & 0.1 & 0.1 & 80 & \\
\hline & 15 & 7.8 & 0.0 & 0.0 & 0.7 & 12.0 & 0.0 & 0.1 & 71.0 & 0.7 & 0.1 & 0.2 & 65 & \\
\hline \multirow[t]{3}{*}{9} & 5 & 6.2 & 0.0 & 0.0 & 0.7 & 13.9 & 0.0 & 0.1 & 69.5 & 0.5 & 0.1 & 0.1 & 120 & \multirow{3}{*}{$\begin{array}{l}\text { Benzene, toluene and the } \\
\text { compounds having molecular } \\
\text { weight } 96,82 \text { and } 56 \text { are } \\
\text { detected in small amount }\end{array}$} \\
\hline & 10 & 10.2 & 0.0 & 0.0 & 0.7 & 9.8 & 0.1 & 0.1 & 71.0 & 0.8 & 0.2 & 0.2 & 120 & \\
\hline & 15 & 8.8 & 0.0 & 0.0 & 0.7 & 11.0 & 0.1 & 0.1 & 70.0 & 1.0 & 0.2 & 0.3 & 125 & \\
\hline \multirow[t]{3}{*}{10} & 5 & 3.1 & 0.0 & 0.0 & 0.7 & 17.2 & 0.1 & 0.1 & 69.5 & 0.3 & 0.1 & 0.1 & 40 & \multirow{3}{*}{$\begin{array}{l}\text { Benzane, toluene and the } \\
\text { compounds having molecular } \\
\text { weight } 96,82 \text { and } 56 \text { are } \\
\text { detected in small amount }\end{array}$} \\
\hline & 10 & 3.8 & 0.0 & 0.0 & 0.7 & 15.8 & 0.1 & 0.1 & 69.0 & 0.6 & 0.2 & 0.2 & 45 & \\
\hline & 15 & 4.4 & 0.0 & 0.0 & 0.7 & 14.8 & 0.1 & 0.1 & 70.0 & 0.6 & 0.2 & 0.2 & 60 & \\
\hline
\end{tabular}

Table 4. The components of the combustion gases $(01 / \mathrm{min})$

\begin{tabular}{|c|c|c|c|c|c|c|c|c|c|c|c|c|c|c|}
\hline $\begin{array}{c}\text { Sample } \\
\text { No. }\end{array}$ & $\begin{array}{c}\text { Time } \\
(\min .)\end{array}$ & $\mathrm{CO}_{2}:(\%)$ & $\mathrm{C}_{3} \mathrm{H}_{8}(\%)$ & $\mathrm{C}_{3} \mathrm{H}_{6}(\%)$ & $\operatorname{Ar}(\%)$ & $0_{2}(\%)$ & $\mathrm{C}_{3} \mathrm{H}_{6}(\%)$ & $\mathrm{C}_{2} \mathrm{H}_{4}(\%)$ & $\mathrm{N}_{2}(\%)$ & $\mathrm{C} 0(\%)$ & $\mathrm{CH}_{4}(\%)$ & $\mathrm{H}_{2}(\%)$ & $\mathrm{HCN}\left(\mathrm{PP}_{\mathrm{n}}\right)$ & \\
\hline \multirow[t]{3}{*}{11} & 5 & 5.8 & 0.0 & 0.0 & 0.7 & 14.0 & 0.0 & 0.0 & 71.0 & 0.3 & 0.0 & 0.1 & 40 & \multirow{3}{*}{$\begin{array}{l}\text { Banzene, toluene and the } \\
\text { compounds having molecular } \\
\text { weight } 96.82 \text { and } 56 \text { are } \\
\text { detected in small amount }\end{array}$} \\
\hline & 10 & 8.0 & 0.0 & 0.0 & 0.7 & 11.6 & 0.0 & 0.1 & 70.0 & 0.5 & 0.3 & 0.2 & 45 & \\
\hline & 15 & 9.0 & 0.0 & 0.0 & 0.7 & 10.4 & 0.0 & 0.1 & 70.0 & 0.6 & 0.1 & 0.2 & 50 & \\
\hline \multirow[t]{3}{*}{12} & 5 & 3.6 & 0.0 & 0.0 & 0.7 & 16.2 & 0.1 & 0.1 & 70.0 & 0.3 & 0.1 & 0.1 & 50 & \multirow{3}{*}{$"$} \\
\hline & 10 & 5.6 & 0.0 & 0.0 & 0.7 & 14.1 & 0.1 & 0.1 & 70.2 & 0.6 & 0.1 & 0.2 & 60 & \\
\hline & 15 & 5.2 & 0.0 & 0.0 & 0.7 & 14.6 & 0.1 & 0.1 & 70.0 & 0.5 & 0.1 & 0.2 & 70 & \\
\hline \multirow[t]{3}{*}{13} & 5 & 4.0 & 0.0 & 0.0 & 0.7 & 15.8 & 0.0 & 0.0 & 69.5 & 0.2 & 0.1 & 0.1 & 65 & \multirow{3}{*}{ " } \\
\hline & 10 & 5.0 & 0.0 & 0.0 & 0.7 & 14.8 & 0.1 & 0.1 & 70.0 & 0.4 & 0.2 & 0.2 & 85 & \\
\hline & 15 & 5.9 & 0.0 & 0.0 & 0.7 & 14.4 & 0.1 & 0.1 & 71.0 & 0.6 & 0.3 & 0.2 & 110 & \\
\hline
\end{tabular}


The Study of Pyrolytic Products of Polymer Materials by Mass Spectroscopy (VII)

The Combustion Gass from Resin Laminated Board in Fire

Table 5. The components of the combustion gases $(1 \mathrm{l} / \mathrm{min})$

\begin{tabular}{|c|c|c|c|c|c|c|c|c|c|c|c|c|c|c|}
\hline $\begin{array}{c}\text { Sample } \\
\text { No. }\end{array}$ & $\begin{array}{c}\text { Time } \\
\text { (min.! }\end{array}$ & $\mathrm{CO}_{2}(\%)$ & $\mathrm{C}_{3} \mathrm{H}_{\mathbf{9}}(\%)$ & $\mathrm{C}_{3} \mathrm{H}_{6}(\%)$ & $\operatorname{Ar}(\%)$ & $0,(\%)$ & $\mathrm{C}_{2} \mathrm{H}_{6}(\%)$ & $\mathrm{C}_{2} \mathrm{H}_{4}(\%)$ & $\mathrm{N}_{2}(\%)$ & $\mathrm{CO}(\%)$ & $\mathrm{CH}_{4}(\%)$ & $\mathrm{H}_{3}(\%)$ & HCN $\left(\mathrm{PP}_{\mathrm{w}}\right)$ & \\
\hline \multirow[t]{3}{*}{1} & 5 & 7.3 & 0.0 & 0.0 & 0.6 & 11.9 & 0.0 & 0.0 & 69.5 & 0.2 & 0.1 & 0.1 & 90 & \multirow{3}{*}{$\begin{array}{l}\text { Benzene and toluene are } \\
\text { detected in small } \\
\text { amount }\end{array}$} \\
\hline & 10 & 7.8 & 0.0 & 0.0 & 0.6 & 11.3 & 0.0 & 0.0 & 73.0 & 0.8 & 0.1 & 0.2 & 80 & \\
\hline & 15 & $7: 4$ & 0.0 & 0.0 & 0.6 & 11.8 & 0.0 & 0.0 & 70.0 & 0.9 & 0.2 & 0.3 & 65 & \\
\hline \multirow[t]{3}{*}{2} & 5 & 5.6 & 0.0 & 0.0 & 0.6 & 13.8 & 0.0 & 0.0 & 69.5 & 0.6 & 0.1 & 0.2 & 95 & \multirow{3}{*}{ " } \\
\hline & 10 & 8.9 & 0.0 & 0.0 & 0.6 & 10.7 & .0 .0 & 0.1 & 69.0 & 1.0 & 0.1 & 0.2 & 80 & \\
\hline & 15 & 8.7 & 0.0 & 0.0 & 0.7 & 10.3 & 0.0 & 0.1 & 70.0 & 1.0 & 0.1 & 0.2 & 70 & \\
\hline \multirow[t]{3}{*}{3} & 5 & 3.4 & 0.0 & 0.0 & 0.7 & 16.2 & 0.0 & 0.1 & 69.5 & 0.6 & 0.1 & 0.1 & 50 & \multirow{3}{*}{ " } \\
\hline & 10 & 4.3 & 0.0 & 0.0 & 0.7 & 15,5 & 0.0 & 0.1 & 69.8 & 0.4 & 0.2 & 0.2 & 70 & \\
\hline & 15 & 4.4 & 0.0 & 0.0 & 0.7 & 15.2 & 0.0 & 0.1 & 69.5 & 0.4 & 0.2 & 0.3 & 90 & \\
\hline \multirow[t]{3}{*}{4} & 5 & 7.2 & 0.0 & 0.0 & 0.6 & 12.8 & 0.0 & 0.1 & 68.0 & 0.7 & 0.3 & 0.2 & 120 & \multirow{3}{*}{ " } \\
\hline & 10 & 9.0 & 0.0 & 0.0 & 0.6 & 10.8 & 0.0 & 0.1 & 67.0 & 0.8 & 0.3 & 0.2 & 100 & \\
\hline & 15 & 9.2 & 0.0 & 0.0 & 0.6 & 10.0 & 0.0 & 0.1 & 69.0 & 0.8 & 0.2 & 0.2 & 115 & \\
\hline \multirow[t]{3}{*}{5} & 5 & 1.6 & 0.0 & 0.0 & 0.6 & 18.2 & 0.0 & 0.1 & 68.0 & 0.4 & 0.1 & 0.1 & 65 & \multirow{3}{*}{$\begin{array}{l}\text { Benzene, toluene and the } \\
\text { compoinds having moleaulor } \\
\text { weight } 56,66 \text { and } 82 \text { are } \\
\text { detected in small amount. }\end{array}$} \\
\hline & 10 & 6.5 & 0.0 & 0.0 & 0.6 & 13.1 & 0.0 & 0.1 & 69.0 & 0.4 & 0.1 & 0.2 & 70 & \\
\hline & 15 & 8.1 & 0.0 & 0.0 & 0.7 & 12.3 & 0.0 & 0.1 & 69.0 & 0.6 & 0.1 & 0.2 & 75 & \\
\hline
\end{tabular}

Table 6. The components of the combustion gases $(1 \mathrm{l} / \mathrm{min})$

\begin{tabular}{|c|c|c|c|c|c|c|c|c|c|c|c|c|c|c|}
\hline $\begin{array}{c}\text { Sample } \\
\text { No. }\end{array}$ & $\begin{array}{l}\text { Time } \\
\text { (min.) }\end{array}$ & $\mathrm{CO}_{2}(\%)$ & $\mathrm{C}_{3} \mathrm{H}_{8}(\%)$ & $\mathrm{C}_{3} \mathrm{H}_{6}(\%)$ & $\operatorname{Ar}(\%)$ & $0_{2}(\%)$ & $\mathrm{C}_{2} \mathrm{H}_{6}(\%)$ & $\mathrm{C}_{2} \mathrm{H}_{4}(\%)$ & $\mathrm{N}_{2}(\%)$ & $\mathrm{CO}(\%)$ & $\mathrm{CH}_{4}(\%)$ & $\mathrm{H}_{2}(\%)$ & $\mathrm{HCN}\left(\mathrm{PP}_{\mathbf{m}}\right)$ & \\
\hline \multirow[t]{3}{*}{6} & 5 & 2.8 & 0.0 & 0.0 & 0.7 & 17.1 & 0.0 & 0.1 & 70.0 & 0.5 & 0.1 & 0.1 & 115 & \multirow{3}{*}{$\begin{array}{l}\text { Benzene and toluene are } \\
\text { detected in saall } \\
\text { amount }\end{array}$} \\
\hline & 10 & 7.2 & 0.0 & 0.0 & 0.6 & 12.5 & 0.0 & 0.1 & 68.0 & 0.5 & 0.2 & 0.2 & 100 & \\
\hline & 15 & 8.0 & 0.0 & 0.0 & 0.6 & 11.2 & 0.0 & 0.1 & 70.0 & 0.8 & 0.2 & 0.3 & 130 & \\
\hline \multirow[t]{3}{*}{7} & 5 & 2.8 & 0.0 & 0.0 & 0.7 & 17.4 & 0.9 & 0.1 & 70.0 & 0.6 & 0.1 & 0.2 & 25 & \multirow{3}{*}{ " } \\
\hline & 10 & 7.1 & 0.0 & 0.0 & 0.6 & 12.9 & 0.0 & 0.1 & 68.5 & 0.4 & 0.1 & 0.2 & 20 & \\
\hline & 15 & 8.4 & 0.0 & 0.0 & 0.7 & 11.4 & 0.0 & 0.0 & 68.0 & 0.6 & 0.2 & 0.3 & 5 & \\
\hline \multirow[t]{3}{*}{8} & 5 & 8.0 & 0.0 & 0.0 & 0.7 & 12.0 & 0.0 & 0.1 & 70.0 & 0.2 & 0.1 & 0.1 & 115 & \multirow{3}{*}{ " } \\
\hline & 10 & 8.6 & 0.0 & 0.0 & 0.6 & 11.0 & 0.0 & 0.1 & 68.0 & 0.7 & 0,3 & 0.4 & 80 & \\
\hline & 15 & 8.6 & 0 & 0.0 & 0.7 & 11.0 & 0.0 & 0.1 & 69.0 & 0.8 & 0.3 & 0.4 & 60 & \\
\hline \multirow[t]{3}{*}{9} & 5 & 3.0 & 0.0 & 0.0 & 0.6 & 16.8 & 0.0 & 0.0 & 69.5 & 0.2 & 0.0 & 0.1 & 55 & \multirow{3}{*}{ " } \\
\hline & 10 & 7.6 & 0.0 & 0.0 & 0.7 & 12.2 & 0.0 & 0.0 & 71.0 & 0.5 & 0.1 & 0.1 & & \\
\hline & 15 & 7.6 & 00 & 0.0 & 0.6 & 12.0 & 0.0 & 0.0 & 70.0 & 0.6 & 0.1 & 0.2 & 90 & \\
\hline \multirow[t]{3}{*}{10} & 5 & 1.4 & 0.0 & 0.0 & 0.7 & 18.2 & 0.0 & 0.0 & 69.0 & 0.8 & 0.1 & 0.2 & 20 & \multirow{3}{*}{ " } \\
\hline & 10 & 2.4 & 0.0 & 0.0 & 0.7 & 17.2 & 0.0 & 0.0 & 69.0 & 1.4 & 0.2 & 0.3 & & \\
\hline & 15 & 3.6 & 0.0 & 0.0 & 0.6 & 16.0 & 0.0 & 0.0 & 68.0 & 0.8 & 0.2 & 0.3 & 40 & \\
\hline
\end{tabular}




\section{K. Hamano and K. Hiramatsu}

Table 7. The components of the combustion gases $(11 / \mathrm{min})$

\begin{tabular}{|c|c|c|c|c|c|c|c|c|c|c|c|c|c|c|}
\hline $\begin{array}{c}\text { Sample } \\
\text { No. }\end{array}$ & $\begin{array}{c}\text { Time } \\
\text { Imin. })\end{array}$ & $\mathrm{CO}_{2}(\%)$ & $\mathrm{C}_{3} \mathrm{H}_{8}(\%)$ & $\mathrm{C}_{3} \mathrm{H}_{6}(\%)$ & $\operatorname{Ar}(\%)$ & $\mathrm{O}_{2}, \frac{1 \%}{\mathrm{~s}}$ & $\mathrm{C}_{2} \mathrm{H}_{6}(\%)$ & $\mathrm{C}_{2} \mathrm{H}_{4}(\%)$ & $N_{2} \mid \% !$ & $(c 0 t \%)$ & $\mathrm{CH}_{4}(\mathrm{O})$ & $\mathrm{H}_{2}(\%)$ & HCVNPPm & \\
\hline \multirow[t]{3}{*}{11} & 5 & 1.4 & 0.0 & 0.0 & 0.6 & 18.4 & 0.0 & 0.1 & 69.0 & 0.4 & 0.1 & 0.1 & 40 & \multirow{3}{*}{$\begin{array}{l}\text { Benzene and toluene are } \\
\text { detected in small amount }\end{array}$} \\
\hline & 10 & 7.4 & 0.0 & 0.0 & 0.6 & 12.4 & 0.0 & 0.1 & 69.0 & 0.4 & 0.1 & 0.2 & 45 & \\
\hline & 15 & 8.8 & 0.0 & 0.0 & 0.7 & 10.8 & 0.0 & 0.1 & 69.0 & 0.6 & 0.2 & 0.3 & 60 & \\
\hline \multirow[t]{3}{*}{12} & 5 & 5.4 & 0.0 & 0.0 & 0.6 & 14.2 & 0.0 & 0.1 & 68.0 & 0.4 & 0.1 & 0.1 & 130 & \multirow{3}{*}{$"$} \\
\hline & 10 & 7.6 & 0.0 & 0.0 & 0.7 & 12.3 & 0.0 & 0.1 & 68.0 & 0.9 & 0.2 & 0.2 & 110 & \\
\hline & 15 & 7.8 & 0.0 & 0.0 & 0.7 & 11.8 & 0.0 & 0.0 & 70.0 & 0.8 & 0.1 & 0.1 & 100 & \\
\hline \multirow[t]{3}{*}{13} & 5 & 1.8 & 0.0 & 0.0 & 0.7 & 18.0 & 0.0 & 0.0 & 70.0 & 0.5 & 0.1 & 0.2 & 60 & \multirow{3}{*}{ " } \\
\hline & 10 & 4.8 & 0.0 & 0.0 & 0.6 & 15.2 & 0.0 & 0.1 & 68.0 & 0.4 & 0.2 & 0.2 & 80 & \\
\hline & 15 & 5.7 & 0.0 & 0.0 & 0.6 & 14.1 & 0.0 & 0.0 & 69.0 & 0.6 & 0.1 & 0.2 & 105 & \\
\hline
\end{tabular}

Table 8 . The components of the combustion gases $(51 / \mathrm{min})$

\begin{tabular}{|c|c|c|c|c|c|c|c|c|c|c|c|c|c|c|}
\hline $\begin{array}{c}\text { Sample } \\
\mathrm{V}_{0}^{\prime} .\end{array}$ & $\begin{array}{l}\text { Time } \\
(\text { min. })\end{array}$ & $\mathrm{CO}_{2} \cdot \%^{\prime}$ & $\mathrm{CO}_{2}{ }^{\prime} \%$; & $\mathrm{C}_{3} \mathrm{H}_{6}(\%)$ & $\operatorname{Ar}(\%)$ & $\mathrm{O}_{2}(\%)$ & $\mathrm{C}_{2} \mathrm{H}_{6}(\%)$ & $\mathrm{C}_{2} \mathrm{H}_{4}(\%)$ & $\mathrm{N}_{2}(\%)$ & $\mathrm{CO}(\%)$ & $\left.\mathrm{CH}_{4} \cdot \%\right\}$ & $\mathrm{H}_{2}(\%)$ & $\mathrm{HCN}(\mathrm{PPm})$ & \\
\hline \multirow[t]{3}{*}{1} & 5 & 1.0 & 0.0 & 0.0 & 0.7 & 19.2 & 0.0 & 0.0 & 68.0 & 0.6 & 0.1 & 0.1 & 80 & \multirow{3}{*}{$\begin{array}{l}\text { Benzene. and toluene are } \\
\text { detected in smmall } \\
\text { amount }\end{array}$} \\
\hline & 10 & 1.8 & 0.0 & 0.0 & 0.6 & 18.1 & 0.0 & 0.0 & 68.0 & 0.9 & 0.2 & 0.2 & 70 & \\
\hline & 15 & 5.8 & 0.0 & 0.0 & 0.6 & 14.2 & 0.0 & 0.0 & 67.5 & 0.7 & 0.1 & 0.2 & 60 & \\
\hline \multirow[t]{3}{*}{2} & 5 & 5.0 & 0.0 & 0.0 & 0.6 & 15.0 & 0.0 & 0.0 & 66.0 & 0.8 & 0.0 & 0.1 & 85 & \multirow{3}{*}{ " } \\
\hline & 10 & 7.4 & 0.0 & 0.0 & 0.6 & 12.4 & 0.0 & 0.0 & 68.0 & 0.8 & 0.0 & 0.1 & 70 & \\
\hline & 15 & 7.6 & 0.0 & 0.0 & 0.6 & 11.9 & 0.0 & 0.0 & 67.0 & 0.7 & 0.1 & 0.1 & 65 & \\
\hline \multirow[t]{3}{*}{3} & 5 & 0.8 & 0.0 & 0.0 & 0.6 & 19.4 & 0.0 & 0.0 & 67.0 & 0.2 & 0.0 & 0.2 & 40 & \multirow{3}{*}{ " } \\
\hline & 10 & 2.0 & 0.0 & 0.0 & 0.6 & 17.8 & 0.0 & 0.0 & 68.0 & 0.6 & 0.1 & 0.2 & 65 & \\
\hline & 15 & 3.6 & 0.0 & 0.0 & 0.6 & 16.0 & 0.0 & 0.0 & 67.0 & 0.6 & 0.1 & 0.2 & 80 & \\
\hline \multirow[t]{3}{*}{4} & 5 & 1.8 & 0.0 & 0.0 & 0.6 & 18.3 & 0.0 & 0.0 & 67.0 & 0.4 & 0.1 & 0.1 & 95 & \multirow{3}{*}{ " } \\
\hline & 10 & 6.2 & 0.0 & 0.0 & 0.6 & 13.6 & 0.0 & 0.0 & 67.0 & 0.4 & 0.1 & 0.2 & 90 & \\
\hline & 15 & 6.6 & 0.0 & 0.0 & 0.6 & 12.8 & 0.0 & 0.0 & 67.0 & 0.6 & 0.1 & 0.1 & 90 & \\
\hline \multirow[t]{3}{*}{5} & 5 & 4.0 & 0.0 & 0.0 & 0.6 & 15.8 & 0.0 & 0.0 & 67.0 & 0.5 & 0.0 & 0.1 & 40 & \multirow{3}{*}{ " } \\
\hline & 10 & 6.2 & 0.0 & 0.0 & 0.6 & 13.6 & 0.0 & 0.0 & 67.0 & 0.4 & 0.0 & 0.1 & 45 & \\
\hline & 15 & 7.2 & 0.0 & 0.0 & 0.6 & 11.6 & 0.0 & 0.1 & 67.0 & 1.0 & 0.1 & 0.2 & 50 & \\
\hline
\end{tabular}


The Study of Pyrolytic Products of Polymer Materials by Mass Spectroscopy (VII)

The Combustion Gass from Resin Laminated Board in Fire

Table 9. The compnnents of the combustion gases $(51 / \mathrm{min})$

\begin{tabular}{|c|c|c|c|c|c|c|c|c|c|c|c|c|c|c|}
\hline $\begin{array}{c}\text { Sample } \\
\text { No. }\end{array}$ & $\begin{array}{c}\text { Time } \\
(\min .) \\
T\end{array}$ & $\mathrm{CO}_{2}(\%)$ & $\mathrm{C}_{3} \mathrm{H}_{6}(\%)$ & $\mathrm{C}_{3} \mathrm{H}_{6}(\%)$ & $\operatorname{Ar}(\%)$ & $0_{2}(\%)$ & $\mathrm{C}_{2} \mathrm{H}_{6}(\%)$ & $\mathrm{C}_{2} \mathrm{H}_{4}(\%)$ & $\mathrm{N}_{2}(\%)$ & $\mathrm{CO}(\%)$ & $\mathrm{CH}_{4}(\%)$ & $\mathrm{H}_{2}(\%)$ & $\mathrm{HCN}\left(\mathrm{PP}_{\mathrm{m}}\right)$ & \\
\hline \multirow[t]{3}{*}{6} & 5 & 7.0 & 0.0 & 0.0 & 0.7 & 13.1 & 0.0 & 0.1 & 68.0 & 0.6 & 0.1 & 0.1 & 80 & \multirow{3}{*}{$\begin{array}{l}\text { Benzene and toluene are } \\
\text { detected in small } \\
\text { amount }\end{array}$} \\
\hline & 10 & 9.0 & 0.0 & 0.0 & 0.6 & 10.8 & 0.0 & 0.1 & 67.0 & 0.7 & 0.2 & 0.2 & 90 & \\
\hline & 15 & 10.2 & 0.0 & 0.0 & 0.6 & 9.4 & 0.0 & 0.1 & 67.0 & 0.7 & 0.2 & 0.3 & 95 & \\
\hline \multirow[t]{3}{*}{7} & 5 & 1.3 & 0.0 & 0.0 & 0.6 & 19.3 & 0.0 & 0.0 & 67.0 & 0.4 & 0.1 & 0.3 & 5 & \multirow{3}{*}{ " } \\
\hline & 10 & 5.4 & 0.0 & 0.0 & 0.6 & 14.8 & 0.0 & 0.0 & 68.0 & 0.3 & 0.1 & 0.2 & 3 & \\
\hline & 15 & 6.6 & 0.0 & 0.0 & 0.6 & 13.4 & 0.0 & 0.0 & 69.0 & 0.2 & 0.3 & 0.4 & 2 & \\
\hline \multirow[t]{3}{*}{8} & 5 & 4.6 & 0.0 & 0.0 & 0.6 & 15.8 & 0.0 & 0.1 & 69.0 & 0.2 & 0.1 & 0.1 & 45 & \multirow{3}{*}{ " } \\
\hline & 10 & 5.8 & 0.0 & 0.0 & 0.6 & 14.2 & 0.0 & 0.0 & 69.0 & 0.4 & 0.1 & 0.2 & 40 & \\
\hline & 15 & 4.8 & 0.0 & 0.0 & 0.6 & 15.0 & 0.0 & 0.0 & 69.0 & 0.2 & 0.1 & 0.2 & 40 & \\
\hline \multirow[t]{3}{*}{9} & 5 & 1.2 & 0.0 & 0.0 & 0.6 & 19.2 & 0.0 & 0.0 & 68.0 & 0.2 & 0.1 & 0.1 & 50 & \multirow{3}{*}{ " } \\
\hline & 10 & 4.6 & 0.0 & 0.0 & 0.7 & 15.6 & 0.0 & 0.0 & 70.0 & 0.2 & 0.1 & 0.2 & 60 & \\
\hline & 15 & 4.8 & 0.0 & 0.0 & 0.6 & 15.2 & 0.0 & 0.0 & 68.0 & 0.6 & 0.1 & 0.2 & 65 & \\
\hline \multirow[t]{3}{*}{10} & 5 & 0.6 & 0.0 & 0.0 & 0.7 & 19.6 & 0.0 & 0.0 & 71.0 & 0.4 & 0.0 & 0.1 & 15 & \multirow{3}{*}{ " } \\
\hline & 10 & 1.6 & 0.0 & 0.0 & 0.6 & 18.8 & 0.0 & 0.0 & 70.0 & 0.3 & 0.1 & 0.2 & 20 & \\
\hline & 15 & 2.2 & 0.0 & 0.0 & 0.7 & 18.0 & 0.0 & 0.0 & 68.0 & 0.4 & 0.1 & 0.3 & 35 & \\
\hline
\end{tabular}

Table 10. The components of the combustion gases $(5 \mathrm{l} / \mathrm{min})$

\begin{tabular}{|c|c|c|c|c|c|c|c|c|c|c|c|c|c|c|}
\hline $\begin{array}{c}\text { Sample } \\
\text { No. }\end{array}$ & $\begin{array}{c}\text { Time } \\
(\mathrm{min} .)\end{array}$ & $\mathrm{CO}_{2}(\%)$ & $\mathrm{C}_{3} \mathrm{H}_{6}(\%)$ & $\mathrm{C}_{3} \mathrm{H}_{6}(\%)$ & $\operatorname{Ar}(\%)$ & $0_{2}(\%)$ & $\mathrm{C}_{2} \mathrm{H}_{6}(\%)$ & $\mathrm{C}_{2} \mathrm{H}_{4}(\%)$ & $\mathrm{N}_{2}(\%)$ & $\mathrm{CO}(\%)$ & $\mathrm{CH}_{4}(\%)$ & $\mathrm{H}_{2}(\%)$ & $\operatorname{HCN}\left(\mathrm{PPm}_{\mathrm{m}}\right)$ & \\
\hline \multirow[t]{3}{*}{11} & 5 & 4.0 & 0.0 & 0.0 & 0.6 & 16.0 & 0.0 & 0.0 & 69.0 & 0.2 & 0.0 & 0.1 & 8 & \multirow{3}{*}{$\begin{array}{l}\text { Benzene and toluene are } \\
\text { detected in small } \\
\text { amount }\end{array}$} \\
\hline & 10 & 6.6 & 0.0 & 0.0 & 0.6 & 13. 2 & 0.0 & 0.0 & 69.0 & 0.4 & 0.1 & 0.1 & 10 & \\
\hline & 15 & 6.8 & 0.0 & 0.0 & 0.6 & 12.8 & 0.0 & 0.0 & 69.0 & 1,0 & 0.1 & 0.2 & 12 & \\
\hline \multirow[t]{3}{*}{12} & 5 & 3.0 & 0.0 & 0.0 & 0.6 & 16.8 & 0.0 & 0.0 & 69.5 & 0.5 & 0.0 & 0.1 & 60 & \multirow{3}{*}{$"$} \\
\hline & 10 & 5.2 & 0.0 & 0.0 & 0.6 & 14.8 & 0.0 & 0.0 & 68.0 & 0.7 & 0.1 & 0.1 & 50 & \\
\hline & 15 & 5.8 & 0.0 & 0.0 & 0.6 & 14.0 & 0.0 & 0.0 & 68.0 & 0.8 & 0.1 & 0.1 & 35 & \\
\hline \multirow[t]{3}{*}{13} & 5 & 1.4 & 0.0 & 0.0 & 0.6 & 18.6 & 0.0 & 0.0 & 68.0 & 0.6 & 0.1 & 0.1 & 45 & \multirow{3}{*}{$"$} \\
\hline & 10 & 4.4 & 0.0 & 0.0 & 0.6 & 15.6 & 0.0 & 0.0 & 69.0 & 0.8 & 0.1 & 0.1 & 60 & \\
\hline & 15 & 4.8 & 0.0 & 0.0 & 0.6 & 14.9 & 0.0 & 0.0 & 69.0 & 0.7 & 0.1 & 0.1 & 100 & \\
\hline
\end{tabular}




\section{K. Hamano and K. Hiramatsu}

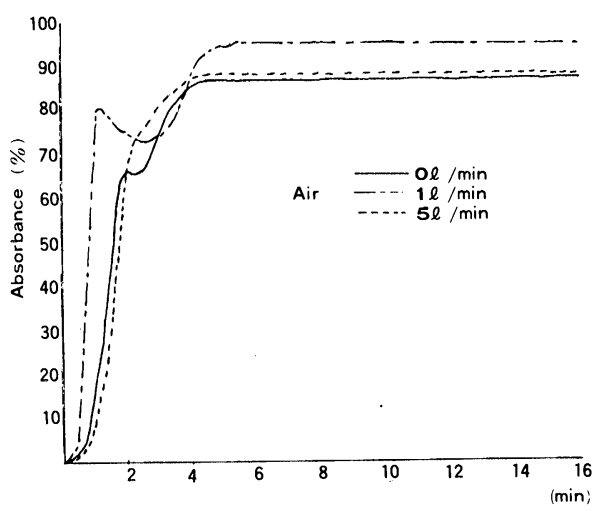

Fig. 15. Concentration of the Smoke (Sample No.1)

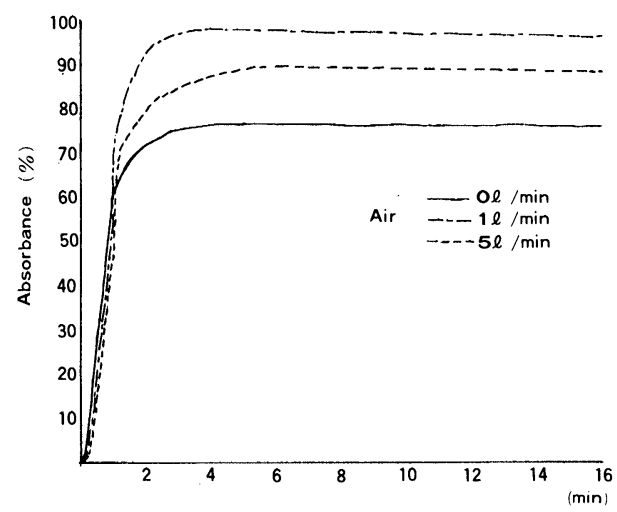

Fig. 17. Concentration of the Smoke (Sample No. 3)

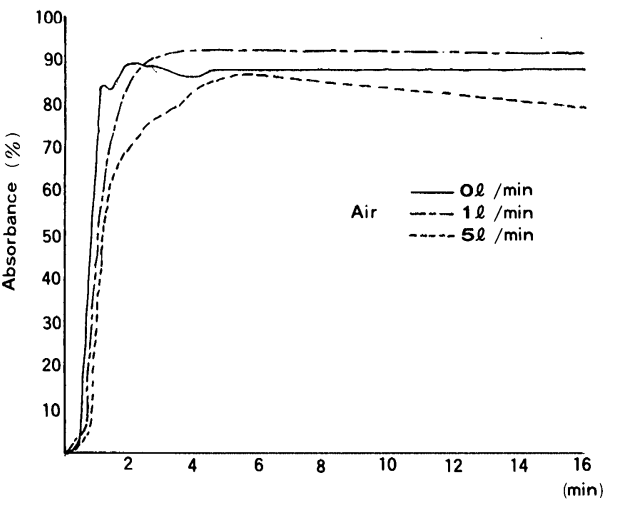

Fig. 19. Concentration of the Smoke (Sample No.5)

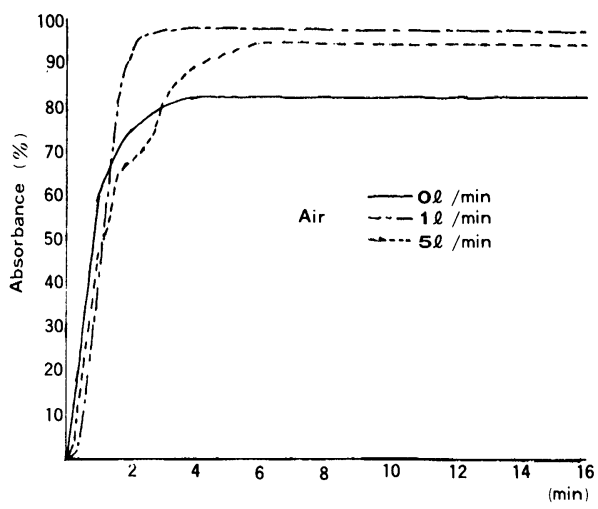

Fig. 16. Concentration of the Smoke (Sample No.2)

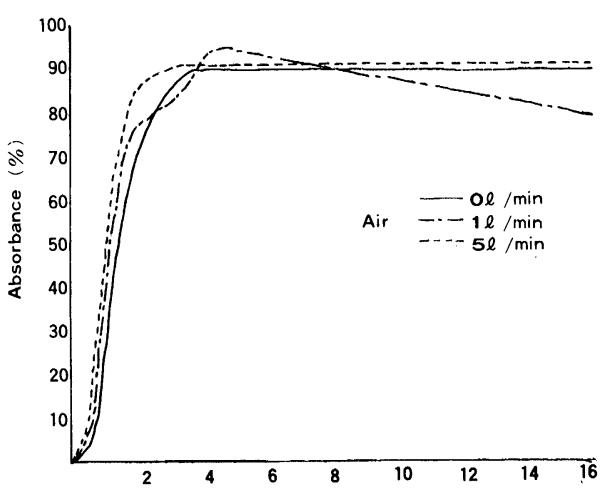

Fig. 18. Concentration of the Smoke (Sample No.4)

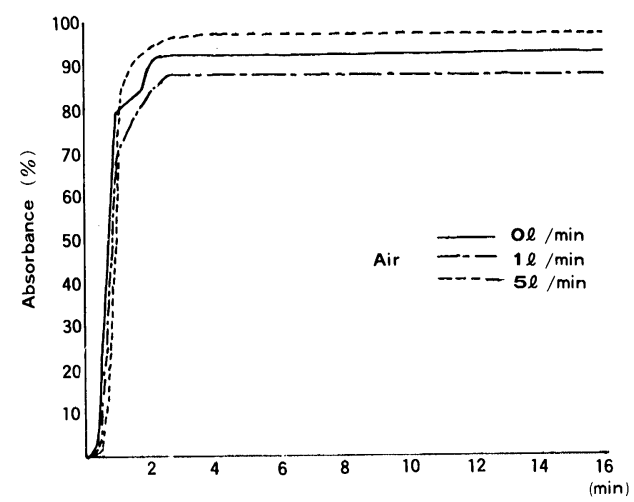

Fig 20. Concentration of the Smoke (Sample No.6) 
The Study of Pyrolytic Products of Polymer Materials by Mass Spectroscopy (VI)

The Combustion Gass from Resin Laminated Board in Fire

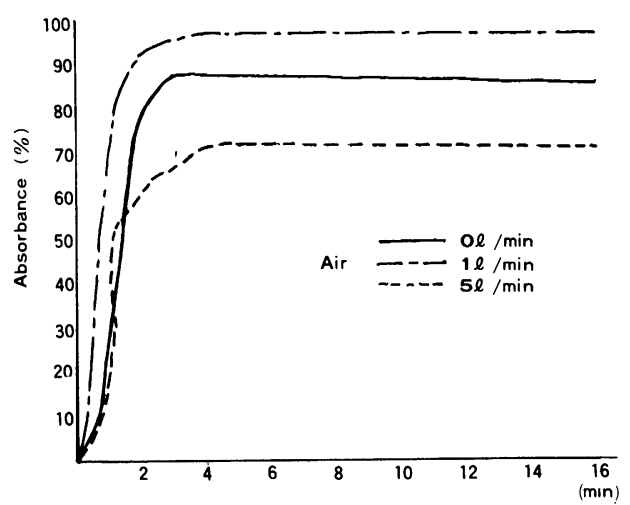

Fig. 21. Concentration of the Smoke (Sample No.7)

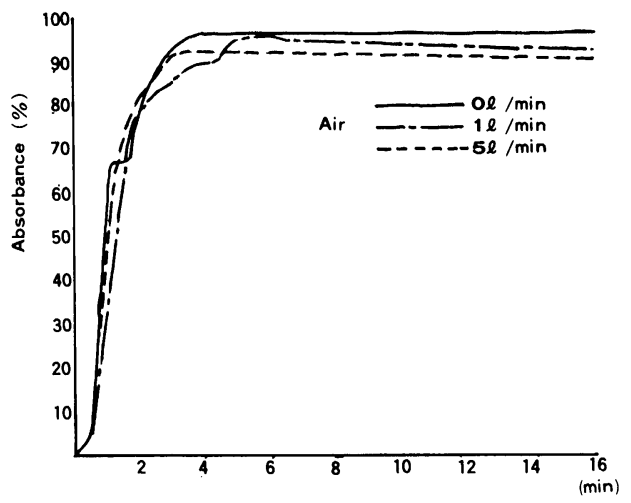

Fig. 23. Concentration of the Smoke (Sample No.9)

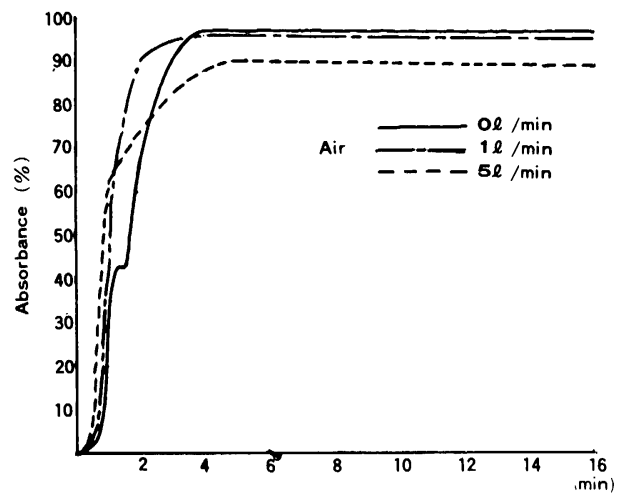

Fig. 25. Concentration of the Smoke (Sample No.11)

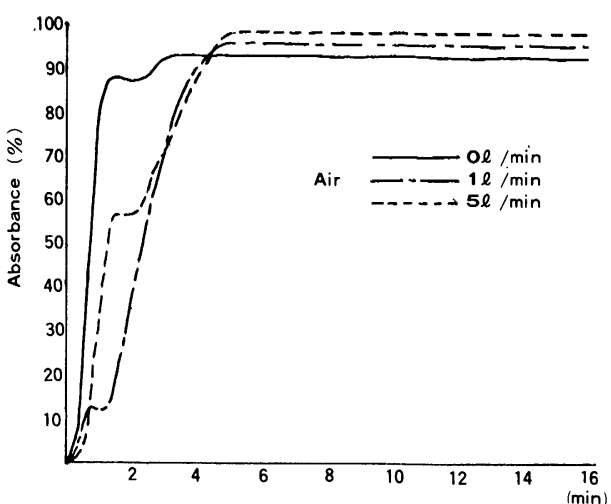

Fig. 22. Concentration of the Smoke (Sample No. 8)

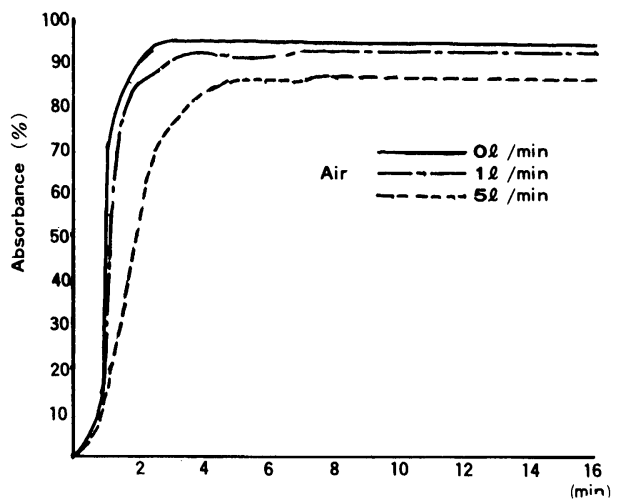

Fig. 24. Concentration of the Smoke (Sample No. 10)

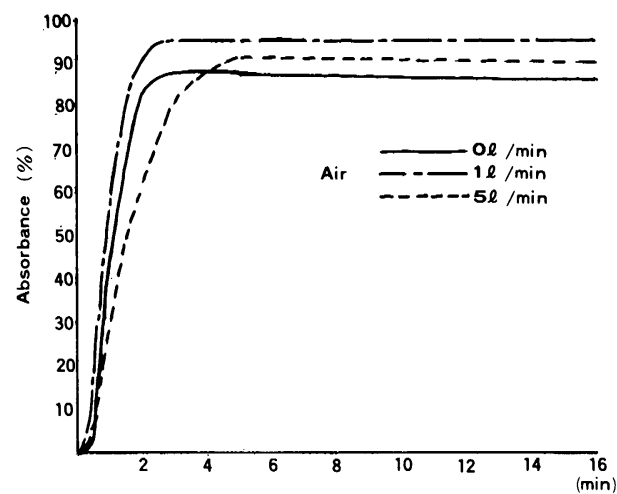

Fig. 26. Concentration of the Smoke (Sample No.12) 


\section{K. Hamano and K. Hiramatsu}

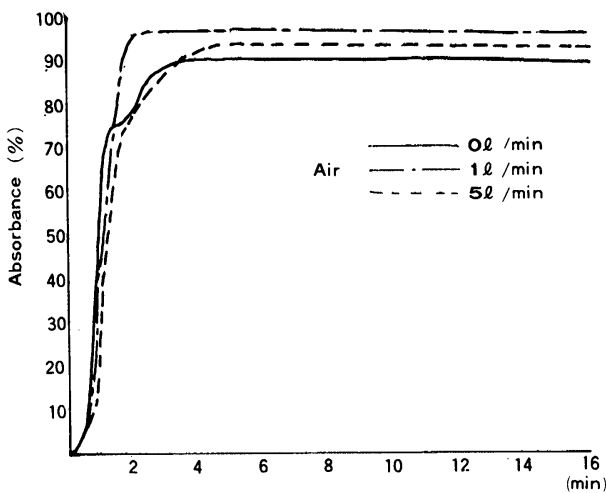

Fig. 27. Concentration of the Smoke (Sample No.13)

\section{Summary}

The components of the conbustion gas of the resin laminated board were determined. The amounts of the hazardous gases by combustion from these materials were found to be much smaller than that from the acrylic fiber.

* Partial result of this paper was presented at the $23 \mathrm{rd}$ Annual meeting of the chemical society of Japan, Tokyo, April, 1970. 